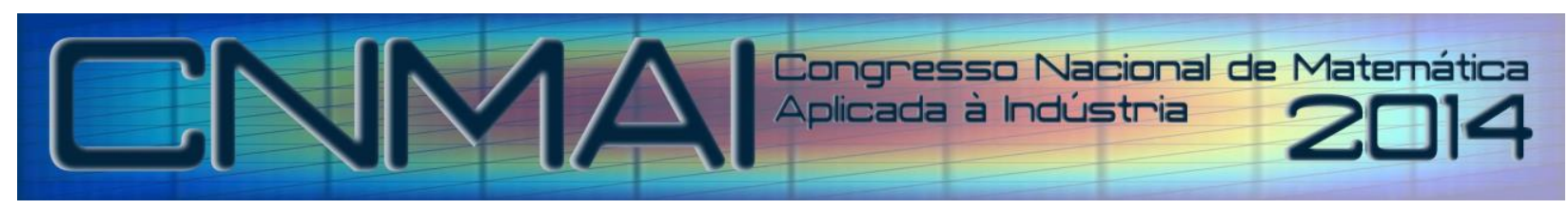

18 a 21 de novembro de 2014, Caldas Novas - Goiás

\title{
USO DE MODELO MATEMÁTICO DE ALOCAÇÃO DE PEÇAS PARA TESTES DE DIFERENTES CENÁRIOS
}

\author{
Tiago dos Santos Almeida, tiago_dosalmeida@hotmail.com ${ }^{1}$ \\ Helio Yochihiro Fuchigami, heliofuchigami@yahoo.com.br ${ }^{2}$ \\ Muris Lage Júnior, muris@ufg.br ${ }^{3}$ \\ Thiago Alves de Queiroz, taq@ufg.br ${ }^{4}$
}

${ }^{1}$ Universidade Federal de Goiás- Departamento de Matemática Industrial, Av. Dr. Lamartine Pinto de Avelar, 1120, Setor Universitário. Bolsista FAPEG.

${ }^{2}$ Universidade Federal de Goiás- Departamento de Matemática Industrial, Av. Dr. Lamartine Pinto de Avelar, 1120, Setor Universitário

${ }^{3}$ Universidade Federal de Goiás- Departamento de Matemática Industrial, Av. Dr. Lamartine Pinto de Avelar, 1120, Setor Universitário

${ }^{4}$ Universidade Federal de Goiás- Departamento de Matemática Industrial, Av. Dr. Lamartine Pinto de Avelar, 1120 , Setor Universitário

\begin{abstract}
Resumo: Com o aumento da competitividade entre as empresas, todos os custos envolvidos em novos projetos têm sidos avaliados, a fim de identificar os possíveis pontos de redução de custos. Desta forma, este trabalho tem o objetivo de apresentar um modelo matemático e realizar testes de diversos cenários, a fim de verificar os impactos no valor ótimo da função objetivo. Com isso, cada instância testada tem o papel de verificar em quais condições o modelo melhor se comporta. Para isso, foram criadas 25 instâncias para a fase de realização de testes, onde foi possível verificar os resultados quando varia-se a demanda, custo de alocação, custo de movimentação, quantidade de embalagens no circuito e diferentes grupos de peças. Para a realização dos testes, utilizou-se o pacote de otimização GUROBI. O modelo matemático utilizado tem como objetivo principal alocar peças em embalagens, à um menor custo total. $O$ custo total envolve os custos de movimentação e estocagem.
\end{abstract}

Palavras-chave: estocagem, otimização, alocação e modelagem inteira.

\section{INTRODUÇÃO}

A partir do aumento da competitividade entre as empresas e as reduções dos recursos financeiros dos novos projetos, faz-se necessário o desenvolvimento de novas metodologias e práticas que possibilitem otimizar os processos a um menor custo. A justificativa para essa tratativa é em função da necessidade de reduzir todos os tipos de desperdícios, além de induzir aos funcionários, sobre a necessidade de criar novas metodologias que utilizem menos recursos e que tenham a mesma ou maior eficiência nas resoluções de problemas.

Nas operações internas de logística é possível detalhar os seus principais custos envolvidos. Os principais custos internos logístico são relacionados as armazenagens das peças no estoque, movimentação interna e embalagens. Neste contexto, o uso de embalagens industriais para a movimentação de peças nos ambientes produtivos tem ganhado destaque em função da sua importância na garantia de um fluxo sincronizado de peças, onde se tenha o ressuprimento de acordo com a demanda, no tempo correto, com a qualidade assegurada e com a máxima funcionalidade. Desta maneira, o desenvolvimento de embalagem tem sido cada vez mais estudado, com o objetivo de fornecer maiores condições para o fluxo logístico, uma vez que a embalagem mal definida poderá acarretar maiores custos logísticos, como o aumento no custo de movimentação, influenciado por uma quantidade de peças/embalagem baixa. Além de, aumentar os riscos de danificar a peça no trajeto do estoque até a montagem final.

Este trabalho tem o objetivo de apresentar as principais características de um modelo matemático que auxilie na determinação do tipo de embalagem para uma determinada peça, a um menor custo total. Desta maneira, serão explicadas as principais variáveis e restrições, necessárias para a escolha do tipo de embalagem, de acordo com a peça. Para isso, 
utilizou-se como base para análise um modelo matemático proposto pelos autores Brizon et al. (2004). Como proposta final, o modelo será testado em 25 instâncias diferentes: variação da demanda, grupo de peças, custo de movimentação, custo de estocagem e quantidade de embalagens disponíveis no circuito.

Na próxima seção serão apresentados os principais conceitos necessários para o entendimento do modelo utilizado, tais como: Custos logísticos, embalagens, problema que envolva custo de movimentação/transporte e custo de estocagem/alocação. Nas demais seções serão detalhadas o modelo e será realizada uma série de testes com diferentes instâncias.

\section{REVISÃO BIBLIOGRÁFICA}

\subsection{Custos logísticos}

Segundo Kussano e Batalha (2012), os custos logísticos podem ser divididos em seis tipos:

- Transportes: custos relacionados aos fretes, perdas, oportunidade de estoques em trânsito e remuneração por estadia.

- Armazenagem: porcentagem de armazenagem nos armazéns.

- Estoque: custos relacionados a oportunidade de estoque nos armazéns.

- Transbordos: taxas cobradas em portos e terminais.

- Portuários: taxas que incidem no uso das infraestruturas portuárias.

- Tributários: taxas que incorrem no transporte interestadual.

Os autores Zeng e Rosseti (2003) detalham os custos logísticos também em seis tipos:

- Transportes: custos incorridos na utilização dos modais de transportes.

- Inventários: custos sobre as operações de realização de inventário.

- Administração: gastos administrativos necessários para a manutenção da logística.

- Alfândegas: taxas relacionadas as movimentações de materiais.

- Riscos e Danos: custos de perdas, avarias e o atraso das mercadorias.

- Manuseio e Embalagem: custos referentes ao manuseio das mercadorias e de embalagens.

\subsection{Embalagens}

Moura e Banzato apud Romano (1996), de acordo com cada área de uma empresa, detalham 13 tipos de conceitos ligados a embalagem. Diante disso, a embalagem tem diferentes significados/funções para cada departamento, tais como: marketing, distribuição física, vendas, finanças, compras, design, jurídica, fornecedores, produção, engenharia de produto, pesquisa e desenvolvimento, garantia da qualidade e engenharia industrial. O último conceito tem maior destaque em função de este trabalho ter o foco em embalagens industriais, ou seja, conceitos que são pertinentes à engenharia industrial no uso de dispositivos de movimentação nos fluxos logísticos.

De uma maneira geral, para Marques (2009), a embalagem tem o poder de alterar os custos de todas as atividades envolvidas, tendo como resultado um grande impacto na relação de produtividade de um sistema logístico. Com isso, têm se os custos de movimentação e armazenagem dependentes da densidade e dimensão da embalagem. Sendo assim, a compreensão do significado, importância e os custos envolvidos a embalagem estão diretamente ligados às características e adaptação de um sistema logístico.

Bowersox e Closs apud Toso (2006) classificam o uso da embalagem em dois tipos, um deles voltados para o consumidor, onde o foco é no marketing, e outro voltado para embalagem industrial, com o foco na logística.

As embalagens são contenedores físicos que tem a função de movimentar, armazenar, transportar, entre outras, peças e componentes de um sistema produtivo. Neste sentido, os custos de embalagens envolvem: matérias-primas como papelão, plástico, madeira, aço, ferro, entre outros tipos, mão de obra e custos relacionados ao desenvolvimento da embalagem. Nas empresas que fornecem embalagens, têm-se os custos de depreciação dos equipamentos, impostos e as margens de lucro inseridas no preço final (BIO et al, 2012).

\subsection{Problemas de custos de Movimentação/Transporte}

Bio et al (2012) definem os custos de movimentação como todas as movimentações associadas na transferência dos materiais dos almoxarifados, áreas de sub montagem, no abastecimento de linhas produtivas e toda transferência de produtos para armazenagem. Desta forma, seus custos são relacionados com a mão de obra envolvida nesta movimentação, bem como a sua manutenção e depreciação sobre os equipamentos utilizados para estas atividades.

No Artigo intitulado "Modelo de Programação quadrática para análise da movimentação logística e comercialização da soja" dos autores Yamakami e Ojima (2006), tem como proposta realizar uma análise sobre os métodos de distribuição da soja brasileira, no intuito de reduzir os custos de transportes, pois é o ponto mais importante nesta cadeia produtiva. Neste sentido, os autores propõem uma aplicação de um modelo de equilíbrio espacial de programação quadrática, que resultará em um fluxo de soja dos locais de oferta até as áreas de demanda, sendo associados aos possíveis novos projetos viários. O objetivo principal é maximizar o lucro total. Com isso, o modelo proposto tornou possível a realização de testes de novos cenários. No cenário B proposto pelos autores, reduzindo os custos dos transportes ferroviários e hidroviários 
em $20 \%$, mantendo o custo dos transportes rodoviários, foi possível verificar as reduções nos custos logísticos de movimentação. O modelo proposto tem a possibilidade de ser testados para outros tipos de cenários.

No decorrer do trabalho "Um algoritmo genético híbrido para o planejamento de movimentação de gás da bacia de campos" dos autores Iachan et al. (2005), com o intuito de melhorar a movimentação de gás da bacia de campos, os autores propuseram um algoritmo genético híbrido, com o objetivo de maximizar o lucro nesta movimentação. No trabalho é apresentado um sistema utilizado pela Petrobras/Campos, o sistema PLANGAS, que utiliza modelos matemáticos de otimização linear com base em bancos de dados. O algoritmo genético proposto poderá ser incorporado ao sistema PLANGAS, como um módulo otimizador. Sendo assim, os resultados com o uso deste algoritmo genético no sistema PLANGAS, obtiveram-se resultados satisfatórios, devido a sua precisão e tempo de processamento.

No desenvolvimento do trabalho "Um modelo de fluxo em rede para a logística de movimentação de contêineres", diante da dificuldade com a logística de contêineres vazios, os autores Medeiros et al. (2008) fazem uma análise baseada na ótica de uma companhia de navegação, para propor um modelo matemático que se baseia em um fluxo em redes multiprodutos, com as suas restrições práticas e com instâncias reais, para minimizar o custo total de reposicionamento dos contêineres vazios. Com isso, o resultado obtido com o uso do modelo matemático, atingiu-se resultados satisfatórios, tornando uma possibilidade de apoio para as decisões táticas da empresa.

A proposta da autora Nakamura (2010) no trabalho "Análise da viabilidade da utilização do transporte por cabotagem para movimentação de automóveis novos no Brasil: Um estudo de caso" é realizar uma análise sobre a viabilidade de transportar automóveis novos por meio da cabotagem, que significa realizar o transporte através da costa marítima brasileira, como alternativa ao modal rodoviário (mais comum). Desta forma, elaborou-se um modelo matemático de transporte, com o uso da programação linear. Neste sentido, o método foi divido em dois tipos: o primeiro, busca de valores de transportes para o modal rodoviário e modal marítimo de cabotagem. O segundo, o uso de um modelo de otimização linear de fluxo de custo mínimo Multiproduto. O resultado apresentado é que o uso da cabotagem alinhado com o modal rodoviário têm possíveis ganhos econômico, porém há a necessidade de ter uma série de alterações no cenário político, tributos e práticas empresariais.

Os autores Hino et al. (2005) propõem no artigo "Estudo e aplicação de transporte colaborativo para cargas de grande volume", uma análise e aplicação de um conceito de transporte colaborativo para carregamentos de alto volume no modal rodoviário, com a justificativa de trazer o máximo do aproveitamento entre as operações de transportes. Para isso, foi proposto um modelo heurístico que irá propor uma função de minimização dos custos do frete do sistema, entre uma empresa A que movimenta soja e uma empresa B que movimenta fertilizante. Com isso, foi alcançado o objetivo de reduzir os custos de frente com o uso do transporte colaborativo.

\subsection{Problemas de custos de Estocagem/Alocação}

O principal objetivo dos autores Pinto et al (2005) do trabalho "Um modelo de programação matemática para alocação estática de caminhões visando ao atendimento de metas de produção e qualidade", é criar um modelo de programação matemática para determinar o ritmo de lavra para cada tipo de frente. Este tipo de problema é conhecido como mistura ou blendagem. Desta forma, o problema leva em consideração a quantidade de equipamentos disponíveis para cada operação e quantidade de caminhões utilizados no transporte. Ou seja, o modelo irá buscar otimizar os seus ganhos operacionais com melhor aproveitamento dos seus recursos. No resultado alcançando foi possível atingir ganhos operacionais com um melhor aproveitamento dos recursos. Entretanto, para alcançar o objetivo, foi necessário adicionar 1 caminhão para atingir as metas, o que reduziu a produtividade cerca de 5,86\%.

No trabalho sobre "Modelos matemáticos para a alocação de peças a embalagens no abastecimento de linhas de montagem" dos autores Brizon et al (2004), o problema estudado consiste em determinar para cada tipo de peça, qual embalagem que atendam um fluxo logístico a um menor custo de movimentação e de estocagem, a fim de reduzir a função objetiva de minimização dos custos totais. Para isso, os autores analisaram as variáveis e restrições do problema e definiram dois tipos de modelos matemáticos, uma para uma demanda uniforme, outro para demanda variável. Para a resolução do problema, foi utilizado o CPLEX.

No decorrer do artigo "Modelo integrado de alocação e movimentação de contêineres vazios e cheio" dos autores Borenstein et al. (2005), justificado por um típico desequilíbrio nas quantidades de contêineres utilizados na exportação e importação, onde é possível identificar diversos contêineres vazios e não utilizados da maneira correta. Desta forma, buscou-se definir um modelo de rede para auxiliar na alocação de contêineres vazios e também auxiliar nas operações de transbordo. Apesar de bastante complexo modelar um problema de alocação de contêineres que envolvam dois aspectos importantes, como: otimização do tempo e nível de serviço, o modelo proposto mostrou resultados eficientes.

\section{MOTIVAÇÃO PRÁTICA PARA O PROBLEMA}

A justificativa principal para o aprofundamento em estudos de determinação do tipo de embalagem utilizar para cada peça está diretamente ligada à importância que as embalagens têm para o funcionamento de um sistema logístico. O papel da embalagem tem ganhado destaque em função da oportunidade de melhorias nos custos logísticos, utilizando embalagens mais adequadas ao processo, que permitam o envio de uma quantidade ideal de peças, com a garantia da integridade, ergonomia e fluxo. 
Desta forma, o problema estudado tem como o principal objetivo analisar os principais cenários que podem ser testados com um modelo de alocação de peça. Neste sentido, a proposta da fase de realização de teste é englobar diferentes situações, que ocorrem nos interiores de ambientes produtivos.

\section{PROBLEMA}

Para a determinação de uma embalagem para cada tipo de peça a um custo total minimizado, utilizou-se o modelo matemático proposto pelos autores Brizon et al (2004), onde o objetivo proposto no seu respectivo trabalho está alinhado a este. Sendo assim, este trabalho dará continuidade aos estudos de alocação de peças em embalagens. O diferencial deste trabalho é que serão testados diversos cenários, tais como: variação da demanda, quantidade de peças/embalagem, custo de movimentação, custo de estocagem e quantidade de embalagens disponíveis para o processo.

\subsection{Definições das variáveis e parâmetros}

I: número de tipos de peças;

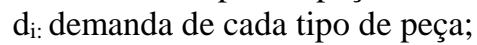

$\mathrm{K}$ : número de embalagens diferentes;

$\mathrm{q}_{\mathrm{ik}}$ : matriz de quantidade de peças/embalagem;

$\mathrm{c}_{\mathrm{i}}$ : custo unitário de estoque;

$\mathrm{b}_{\mathrm{k}}$ : custo fixo de movimentação

lk: limite de embalagens no processo

$\mathrm{W}_{\mathrm{ik}}$ : número de embalagens do tamanho $\mathrm{k}$ em relação a necessidade da peça $\mathrm{i}$;

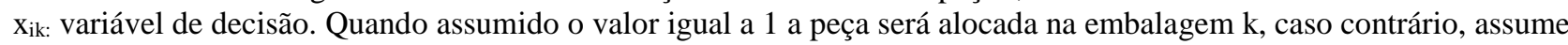
o valor 0 .

\subsection{Função Objetivo}

Minimizar

$$
\sum_{k=1}^{K} \sum_{i=1}^{I}\left(\frac{\text { di.bk }}{\text { qik }}+\frac{\text { ci.qik }}{2}\right) x i k
$$

A função objetivo representado pela Eq.(1) será para minimizar os custos totais, que envolve os custos de movimentação de peças e de alocação.

\subsection{Restrições}

Sujeito à

$$
\begin{array}{rl}
\sum_{k=1}^{K} & x i k=1 \\
& \text { Para todo } \mathrm{i}=1, \ldots, \mathrm{I} \\
\sum_{i=1}^{I} & \text { wik. } x i k \leq \mathrm{k} \\
& \text { Para todo } \mathrm{i}=1, \ldots, \mathrm{I} \\
\mathrm{x}_{\mathrm{ik}} \in & \{0,1\} \\
& \text { Para todo } \mathrm{i}=1, \ldots, \mathrm{I} \text { e } \mathrm{k}=1, \ldots, \mathrm{K}
\end{array}
$$

As restrições utilizadas têm o papel de delimitar para cada tipo de peça uma única embalagem, restrição 1 (eq. 2) além de ter uma restrição para que as quantidades de embalagens necessárias não excedam a quantidade disponível no processo, restrição 2 (eq. 3). Por fim, a restrição 3 (eq.4) tem o papel de indicar para cada tipo de peça qual é a embalagem utilizada.

\section{MÉTODO DE RESOLUÇÃO}

O modelo proposto pelos autores Brizon et al (2004) minimizar os custos de movimentação e os custos de estocagem. Para isso, a análise se baseia nas informações de demanda unitária e a quantidade máxima de peças por modelo de embalagem. Além disso, para que o problema possa ser aplicado em casos reais, foi definido um fator limitante para cada embalagem, ou seja, para cada modelo têm-se um limite de embalagens no circuito logístico.

Para a resolução do algoritmo de alocação de peças, todos os testes e resultados computacionais, apresentados no decorrer deste trabalho, foram realizados em um desktop com processador Intel ® Core ${ }^{\mathrm{TM}}$ i5-4440 (3,1 GHz, cache de $6 \mathrm{Mb})$ e 4GB RAM. O pacote de otimização utilizado é o Gurobi Optimization, Version 5.6. O processo de implementação do algoritmo no GUROBI pode ser resumido em 6 etapas:

- Declarar as variáveis do problema;

- Declaração de leitura de dados de arquivo externo (Exemplo: .txt);

- Declaração das variáveis do problema de otimização;

- Declaração da função objetivo; 
- Declaração das restrições do problema;

- Impressão das variáveis e função objetivo.

A etapa de definição dos dados externos que serão utilizados para o processo de otimização tem um papel fundamental nos resultados do modelo. Neste trabalho, foram construídas 25 instâncias com o objetivo de verificar os diversos comportamentos que o modelo tem a cada variação nos dados. Sendo assim, foram realizados testes com variação na demanda, custos de estocagem, custo de movimentação, capacidade de peças por embalagem, limite de embalagens no circuito, pequenos e grandes dados.

Para a compreensão do funcionamento das instâncias, ou seja, os dados que o modelo irá trabalhar serão detalhados abaixo o significado de valor declarado em relação ao algoritmo utilizado. Desta forma, a $1^{\mathrm{a}}$ instância utilizada no pacote de otimização tem os seguintes dados externos, gerados aleatoriamente:

Tabela 1. Lista dos dados externos da $\mathbf{1}^{\mathrm{a}}$ instância.

\begin{tabular}{|c|c|}
\hline \multicolumn{2}{|c|}{ Lista de dados externos } \\
\hline 10: Quantidade de peças i; & 212 22: Matriz $q_{\text {ik }}$ da capacidade de peças 8 nas embalagens $1,2,3$; \\
\hline 100: Demanda $d_{1}$ da peça 1 ; & 818 28: Matriz $_{\mathrm{ik}}$ da capacidade de peças 9 nas embalagens $1,2,3$; \\
\hline 150: Demanda $d_{2}$ da peça 2 ; & 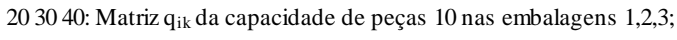 \\
\hline 300: Demanda d da peça 3 ; & 5.1: Custo de estocagem da peça 1 \\
\hline 250: Demanda $\mathrm{d}_{4}$ da peça 4; & 15.3: Custo de estocagem da peça 2; \\
\hline 300: Demanda d $\mathrm{d}_{5}$ da peça 5 ; & 24.5: Custo de estocagem da peça 3; \\
\hline 150: Demanda d 6 da peça 6 ; & 28.6: Custo de estocagem da peça 4; \\
\hline 50: Demanda $d_{7}$ da peça 7 & 63.8: Custo de estocagem da peça 5; \\
\hline 500: Demanda d $d_{8}$ da peça 8 & 58.6: Custo de estocagem da peça 6 ; \\
\hline 80: Demanda d da peça 9; & 14.1: Custo de estocagem da peça 7 ; \\
\hline 160: Demanda $\mathrm{d}_{10}$ da peça 10 ; & 27.7: Custo de estocagem da peça 8 ; \\
\hline 3: Número de embalagens diferentes; & 89.7: Custo de estocagem da peça 9 ; \\
\hline 1020 30: Matriz $q_{i k}$ da capacidade de peças 1 nas embalagens $1,2,3$; & 24.8: Custo de estocagem da peça 10 \\
\hline 2030 40: Matriz $q_{i k}$ da capacidade de peças 2 nas embalagens $1,2,3$; & 15.2: Custo de movimentação da embalagem 1 ; \\
\hline 3040 50: Matriz $q_{i k}$ da capacidade de peças 3 nas embalagens $1,2,3$; & 24.4: Custo de movimentação da embalagem 2; \\
\hline 2030 40: Matriz $q_{i k}$ da capacidade de peças 4 nas embalagens $1,2,3$; & 30.7: Custo de movimentação da embalagem 3 ; \\
\hline 1525 35: Matriz $q_{i k}$ da capacidade de peças 5 nas embalagens $1,2,3$; & 35: Limite de embalagens no circuito do tipo 1 ; \\
\hline 1020 30: Matriz $q_{i k}$ da capacidade de peças 6 nas embalagens $1,2,3$; & 38: Limite de embalagens no circuito do tipo 2; \\
\hline 10 10 20: Matriz $q_{i k}$ da capacidade de peças 7 nas embalagens $1,2,3$; & 40: Limite de embalagens no circuito do tipo 3 ; \\
\hline
\end{tabular}

Por fim, definiu-se que para cada processo de otimização o GUROBI irá escolher automaticamente o algoritmo a ser utilizado, tais como: Simplex primal, Simplex dual, entre outros.

\section{RESULTADOS}

\subsection{Instâncias 1 e 2}

Para verificar o comportamento do modelo matemático, quando alterado as demandas de cada tipo de peça, realizouse teste nas duas primeiras instâncias. Com isso, os dados utilizados para as duas instâncias estão representados na tabela 2. A diferença entre as duas é a variação na demanda. Observa-se que para este teste foram utilizados um grupo de peças 1 , onde não varia a quantidade de peças/embalagem, custo de estocagem, custo de movimentação e quantidade de embalagens disponíveis. Na instância 1, considera-se os dados da demanda 1, já na instância 2 , os dados da demanda 2 .

Tabela 2. Resultados dos testes com as instâncias 1 e 2.

\begin{tabular}{|c|c|c|c|c|c|c|c|}
\hline \multicolumn{8}{|c|}{ INSTAANCIAS 01,02 - GRUPO } \\
\hline Número & Nome da Peça & Demanda 1 & Demanda 2 & \multicolumn{3}{|c|}{ Quantidade de Peças/Embalagem } & Custo de Estocagem \\
\hline 1 & PORCA, M15 & 100 & 150 & 10 & 20 & 30 & 5.1 \\
\hline 2 & PARAFUSO, PERFURANTE & 150 & 100 & 20 & 30 & 40 & 15.3 \\
\hline 3 & BRACKET & 300 & 50 & 30 & 40 & 50 & 24.5 \\
\hline 4 & VOLANTE, CJ & 250 & 130 & 20 & 30 & 40 & 28.6 \\
\hline 5 & SENSOR, HIDRAULICO & 300 & 200 & $\frac{25}{15}$ & 25 & 35 & 63.8 \\
\hline 6 & MÓDULO & 150 & 150 & 10 & 20 & 30 & 58.6 \\
\hline 7 & CHICOTE ELÉTRICO & 50 & 135 & 10 & 10 & 20 & 14.1 \\
\hline 8 & CJ LANTERNA, TRASEIRA & 500 & 150 & 21 & 22 & 2 & 27.7 \\
\hline 9 & CONJUNTO, SETA & 80 & 170 & 81 & 82 & 8 & 89.7 \\
\hline \multirow[t]{3}{*}{10} & & 20 & 30 & 40 & 24.8 \\
\hline & & & & 15.2 & 24.4 & 30,7 & \\
\hline & Quantidade de Em & alagens disp & & 35 & 38 & 40 & \\
\hline
\end{tabular}

Com o pacote de otimização foi possível resolver as instâncias 1 e 2, obtendo os seguintes valores: no primeiro, o tempo de resolução é de 0,003 segundos, com o valor ótimo da função de 2269,618. Na segunda, o tempo de resolução é de 0,002 segundos, com o valor ótimo da função de 1718,873. 


\subsection{Instâncias 3 e 4}

Para a verificação do comportamento do modelo foi alterado o grupo de peças, mantendo a mesma demanda, custo de estocagem, custo de movimentação e limites de embalagens no circuito. Ou seja, alterando o grupo de peças, buscou utilizar peças com dimensões diferentes o que influencia diretamente na quantidade de peças/embalagem. Sendo assim, na instância 3 foi utilizado o grupo de peças 2, com diferentes quantidades/embalagem. Na instância 4 foi utilizado o grupo de peças 3 , com diferentes quantidades/embalagem.

Tabela 3. Resultados dos testes com a instância 3.

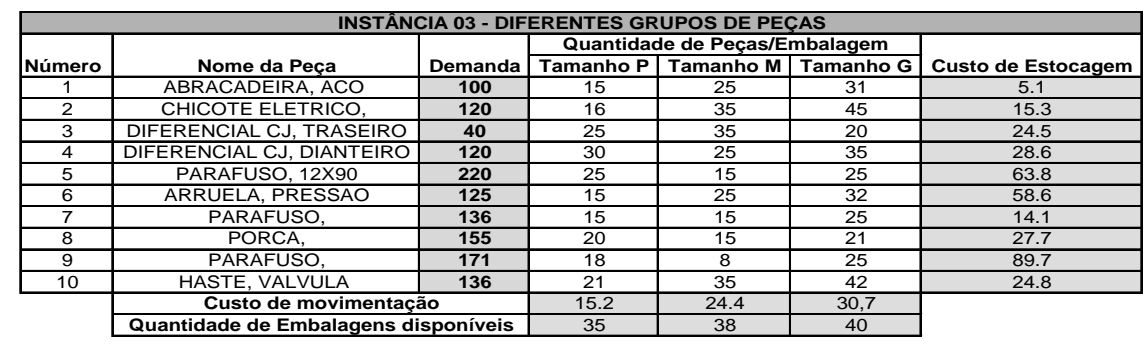

Tabela 4. Resultados dos testes com a instância 4.

\begin{tabular}{|c|c|c|c|c|c|c|}
\hline \multicolumn{7}{|c|}{ INSTÂNCIA 04 - DIFERENTES GRUPOS DE PEÇAS } \\
\hline \multirow[b]{2}{*}{ Número } & \multirow[b]{2}{*}{ Nome da Peça } & \multirow[b]{2}{*}{ Demanda } & \multicolumn{3}{|c|}{ Quantidade de Peças/Embalagem } & \multirow[b]{2}{*}{ Custo de Estocagem } \\
\hline & & & Tamanho P & Tamanho M & Tamanho G & \\
\hline 1 & PARAFUSO $10 \times 35$ & 100 & 12 & 11 & 11 & 5.1 \\
\hline 2 & BARRA CJ, DIRECAO & 120 & 16 & 35 & 15 & 15.3 \\
\hline 3 & PRESILHA, SUPERIOR TERMINAL & 40 & 15 & 15 & 10 & 24.5 \\
\hline 4 & CJ, EIXO CARDAN & 120 & 10 & 15 & 25 & 28.6 \\
\hline$\frac{7}{5}$ & TRANSFERENCIA,CJ. & 220 & 15 & 5 & $\frac{25}{15}$ & 63.8 \\
\hline$\frac{6}{6}$ & TRANSMISSAO CJ, MANUAL & 125 & 5 & 15 & 22 & 58.6 \\
\hline 7 & SUPORTE, TUBO EXAUSTAO & 136 & 15 & 15 & 25 & 14.1 \\
\hline 8 & COXIM, CAIXA TRANSFERENCIA & 155 & 10 & 5 & 11 & 27.7 \\
\hline 9 & COXIM DE BORRACHA & 171 & 8 & 18 & 15 & 89.7 \\
\hline \multirow[t]{3}{*}{10} & PORCA, & 136 & 11 & 25 & 12 & 24.8 \\
\hline & \multicolumn{2}{|c|}{ Custo de movimentação } & 15.2 & 24.4 & 30,7 & \\
\hline & Quantidade de Embalagen & disponíveis & 35 & 38 & 40 & \\
\hline
\end{tabular}

Com a resolução do modelo de acordo com as alterações dos grupos de peças, obtiveram-se os seguintes resultados: instância 3, o tempo de resolução é de 0,003 segundos, com o valor ótimo de 1350,382. Na instância 4, o tempo de resolução é de 0,003 segundos, com o valor ótimo da função de 1934,478.

\subsection{Instâncias 5 e 6}

Nas instâncias 5 e 6 foi considerado o grupo de peças 3, com uma mesma demanda, custo de movimentação, quantidade de peças/embalagem e quantidade de embalagens disponíveis. Entretanto, variaram-se os custos de estocagem para verificar o comportamento do modelo. Para a instância 5 foi utilizado o custo de estocagem 1 , já para a instância 6 , o custo de estocagem 2 .

Tabela 5. Resultados dos testes com as instâncias 5 e 6.

\begin{tabular}{|c|c|c|c|c|c|c|c|}
\hline \multicolumn{8}{|c|}{ INSTÂNCIA 05,06 - GRUPO DE PEÇAS 3 COM DIFERENTES CUSTOS DE ESTOCAGEM } \\
\hline \multirow[b]{2}{*}{ Número } & \multirow[b]{2}{*}{ Nome da Peça } & \multirow[b]{2}{*}{ Demanda } & \multicolumn{3}{|c|}{ Quantidade de Peças/Embalagem } & \multirow[b]{2}{*}{ Custo de Estocagem 1} & \multirow[b]{2}{*}{ Custo de Estocagem 2} \\
\hline & & & Tamanho P & Tamanho M & Tamanho G & & \\
\hline 1 & PARAFUSO $10 \times 35$ & 100 & 12 & 11 & 11 & 5.1 & 15.1 \\
\hline 2 & BARRA CJ, DIRECAO & 120 & 16 & 35 & 15 & 10.3 & 20.3 \\
\hline 3 & PRESILHA, SUPERIOR TERMINAL & 40 & 15 & 15 & 10 & 10.5 & 30.5 \\
\hline 4 & CJ, EIXO CARDAN & 120 & 10 & 15 & 25 & 15.6 & 25.6 \\
\hline 5 & TRANSFERENCIA,CJ. & 220 & 15 & 5 & 15 & 45.8 & 55.8 \\
\hline 6 & TRANSMISSAO CJ, MANUAL & 125 & 5 & 15 & 22 & 37.6 & 57.6 \\
\hline 7 & SUPORTE, TUBO EXAUSTAO & 136 & 15 & 15 & 25 & 18.1 & 28.1 \\
\hline 8 & COXIM, CAIXA TRANSFERENCIA & 155 & 10 & 5 & 11 & 18.7 & 28.7 \\
\hline 9 & COXIM DE BORRACHA & 171 & 8 & 18 & 15 & 32.7 & 62.7 \\
\hline \multirow[t]{3}{*}{10} & PORCA & 136 & 11 & 25 & 12 & 15.8 & 85.8 \\
\hline & \multicolumn{2}{|c|}{ Custo de movimentação } & 15.2 & 24.4 & 30,7 & & \\
\hline & \multicolumn{2}{|c|}{ Quantidade de Embalagens disponíveis } & 35 & 38 & 40 & & \\
\hline
\end{tabular}

Os resultados obtidos para a instância 5 é um tempo de resolução de 0,003 segundos e valor ótimo da função de 1863,478. Para a instância 6 o tempo de resolução também é de 0,003 segundos, com o valor ótimo de 1963,478.

\subsection{Instâncias 7 e 8}

Os testes realizados nas instâncias 7 e 8, tem como o objetivo de variar os custos de movimentação para o mesmo grupo de peças 3. Desta forma, manteve-se a demanda, quantidade de peças/embalagem, custo de estocagem e quantidade de embalagens disponíveis. Na instância 7, utilizou-se do custo de movimentação 1, na instância 8, o custo de movimentação 2 . 
Tabela 6. Resultados dos testes com as instâncias 7 e 8.

\begin{tabular}{|c|c|c|c|c|c|c|}
\hline \multicolumn{7}{|c|}{ INSTÂNCIA 07, 08 - GRUPO DE PEÇAS 3 COM DIFERENTES CUSTOS DE MOVIMENTAÇÃO } \\
\hline \multirow[b]{2}{*}{ Número } & \multirow[b]{2}{*}{ Nome da Peça } & \multirow[b]{2}{*}{ Demanda } & \multicolumn{3}{|c|}{ Quantidade de Peças/Embalagem } & \multirow[b]{2}{*}{ Custo de Estocagem } \\
\hline & & & Tamanho P & Tamanho M & Tamanho G & \\
\hline 1 & PARAFUSO $10 \times 35$ & 100 & 12 & 11 & 11 & 15.1 \\
\hline 2 & BARRA CJ, DIRECAO & 120 & 16 & 35 & 15 & 20.3 \\
\hline 3 & PRESILHA, SUPERIOR TERMINAL & 40 & 15 & 15 & 10 & 30.5 \\
\hline 4 & CJ, EIXO CARDAN & 120 & 10 & 15 & 25 & 25.6 \\
\hline 5 & TRANSFERENCIA,CJ. & 220 & 15 & 5 & 15 & 55.8 \\
\hline 6 & TRANSMISSAO CJ, MANUAL & 125 & 5 & 15 & 22 & 57.6 \\
\hline 7 & SUPORTE, TUBO EXAUSTAO & 136 & 15 & 15 & 25 & 28.1 \\
\hline 8 & COXIM, CAIXA TRANSFERENCIA & 155 & 10 & 5 & 11 & 28.7 \\
\hline 9 & COXIM DE BORRACHA & 171 & 8 & 18 & 15 & 62.7 \\
\hline \multirow[t]{4}{*}{10} & PORCA, & 136 & 11 & 25 & 12 & 85.8 \\
\hline & \multicolumn{2}{|c|}{ Custo de movimentação 1} & 35,2 & 58,4 & 60,7 & \\
\hline & \multicolumn{2}{|c|}{ Custo de movimentação 2} & 65,2 & 98,4 & 70,7 & \\
\hline & \multicolumn{2}{|c|}{ Quantidade de Embalagens disponíveis } & 35 & 38 & 40 & \\
\hline
\end{tabular}

No resultado da instância 7, o tempo de resolução foi de 0,003 segundos, com um valor ótimo da função objetivo de 4031,421. A instância 8, o tempo de resolução foi de 0,001, com o valor ótimo da função objetivo de 5779,114.

\subsection{Instâncias 9 e 10}

Nas instâncias 9 e 10 buscou-se verificar o comportamento do modelo quando alterado a quantidade de embalagens disponíveis no circuito logístico. Desta forma, na instância 9 foi considerado a quantidade de embalagens disponíveis 1. Na instância 10 foi considerado a quantidade de embalagens disponíveis 2 .

Tabela 7. Resultados dos testes com as instâncias 9 e 10.

\begin{tabular}{|c|c|c|c|c|c|c|}
\hline \multicolumn{7}{|r|}{ DISPONIVEIS } \\
\hline \multirow[b]{2}{*}{ Número } & \multirow[b]{2}{*}{ Nome da Peça } & \multirow[b]{2}{*}{ Demanda } & \multicolumn{3}{|c|}{ Quantidade de Peças/Embalagem } & \multirow[b]{2}{*}{ Custo de Estocagem } \\
\hline & & & Tamanho P & Tamanho M & Tamanho G & \\
\hline 1 & PORCA, M15 & 100 & 12 & 11 & 11 & 15.1 \\
\hline 2 & PARAFUSO, PERFURANTE & 120 & 16 & 35 & 15 & 20.3 \\
\hline 3 & BRACKET & 40 & 15 & 15 & 10 & 30.5 \\
\hline 4 & VOLANTE, CJ & 120 & 10 & 15 & 25 & 25.6 \\
\hline 5 & SENSOR, HIDRÁULICO & 220 & 15 & 5 & 15 & 55.8 \\
\hline 6 & MODDULO & 125 & 5 & 15 & 22 & 57.6 \\
\hline 7 & CHICOTE ELÉTRICO & 136 & 15 & 15 & 25 & 28.1 \\
\hline 8 & CJ LANTERNA, TRASEIRA & 155 & 10 & 5 & 11 & 28.7 \\
\hline 9 & CONJUNTO, SETA & 171 & 8 & 18 & 15 & 62.7 \\
\hline \multirow[t]{4}{*}{10} & MÁQUINA DE VIDRO & 136 & 11 & 25 & 12 & 85.8 \\
\hline & \multicolumn{2}{|c|}{ Custo de movimentação } & 65,2 & 98,4 & 70,7 & \\
\hline & \multirow{2}{*}{\multicolumn{2}{|c|}{$\begin{array}{l}\text { Quantidade de Embalagens disponiveis } 1 \\
\text { Quantidade de Embalagens disponiveis } 2\end{array}$}} & 25 & 28 & 30 & \\
\hline & & & 85 & 98 & 50 & \\
\hline
\end{tabular}

Os dados obtidos com essa alteração de quantidade de embalagens disponíveis foram: instância 9, o tempo de resolução foi de 0,002 , com o valor ótimo da função de 5909,434. Na instância 10, o tempo de resolução foi de 0,002 , com o valor ótimo da função objetivo de 5779,114.

\section{ANÁLISE COMPARATIVA DOS DADOS}

Com base nos resultados obtidos no decorrer da fase de experimentação, apresentados no tópico anterior, será possível compreender melhor o funcionamento do modelo matemático proposto pelos autores Brizon et al (2004). Desta forma, ao analisar os resultados obtidos na primeira fase de testes, instância 1 e instância 2, onde houve a variação na demanda de cada item, é possível verificar que na medida que a demanda de cada item é aumentada o valor ótimo da função objetivo também é elevada. A justificativa para este resultado é que com uma demanda alta, com o mesmo custo de movimentação, custo de estocagem e de quantidade de peças/embalagem, aumenta-se a frequência de movimentação para atender a demanda.

Tabela 8. Resultado das análises das instâncias 1 e 2.

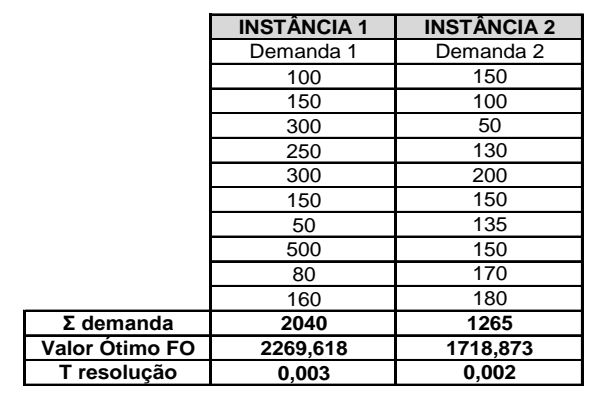


Ao analisar os resultados obtidos nas instâncias 3 e 4, onde utilizando os mesmos parâmetros de demanda, quantidade de embalagens no circuito, custo de movimentação e estocagem, alterando apenas o grupo de peças (2 e 3), verifica-se que com esta alteração a quantidade de peças/embalagem entre os dois grupos de peças, influenciou diretamente no valor ótimo da função objetivo. A justificativa é que quando a quantidade média de peças por embalagem se reduz, aumentase a necessidade de utilizar mais embalagens e têm-se maior movimentação de peças entre o estoque e linha de produção.

Tabela 9. Resultado das análises das instâncias 3 e 4.

\begin{tabular}{|c|c|c|c|c|c|c|}
\hline & \multicolumn{3}{|c|}{ INSTÁNCIA 3} & \multicolumn{3}{|c|}{$\begin{array}{c}\text { INSTÄNCIA 4 } \\
\text { GRUPO DE PECAS } 3\end{array}$} \\
\hline & Quantidad & GRUPO DE PEÇAS 2 & mbalagem & \multicolumn{3}{|c|}{$\begin{array}{c}\text { GRUPO DE PEÇAS } 3 \\
\text { Quantidade de Peças/Embalagem }\end{array}$} \\
\hline & Tamanho P & Tamanho M & Tamanho G & Tamanho P & Tamanho M & Tamanho G \\
\hline & 15 & \begin{tabular}{|l|}
25 \\
\end{tabular} & 31 & 12 & 11 & 11 \\
\hline & 16 & 35 & 45 & 16 & 35 & 15 \\
\hline & 25 & 35 & 20 & 15 & 15 & 10 \\
\hline & 30 & 25 & 35 & 10 & 15 & 25 \\
\hline & 25 & 15 & 25 & 15 & 5 & 15 \\
\hline & 15 & 25 & 32 & 5 & 15 & 22 \\
\hline & 15 & 15 & 25 & 15 & 15 & 25 \\
\hline & 20 & 15 & 21 & 10 & 5 & 11 \\
\hline & 18 & 8 & 25 & 8 & 18 & 15 \\
\hline & 21 & 35 & 42 & 11 & 25 & 12 \\
\hline Valor médio peças/embalagem & \multicolumn{3}{|c|}{24,47} & \multicolumn{3}{|c|}{14,57} \\
\hline \begin{tabular}{|c|} 
Valor Otimo FO \\
\end{tabular} & \multirow{2}{*}{\multicolumn{3}{|c|}{$\begin{array}{c}1350,383 \\
0,003\end{array}$}} & \multirow{2}{*}{\multicolumn{3}{|c|}{$\frac{1934,478}{0,003}$}} \\
\hline T resolução & & & & & & \\
\hline
\end{tabular}

Com os resultados obtidos nos testes com as instâncias 5 e 6, foi possível entender que os custos de estocagem influenciam diretamente no valor ótimo da função objetivo, ou seja, quanto maior o custo médio de estocagem, maior será o valor ótimo obtido da função objetivo.

Tabela 10. Resultado das análises das instâncias 5 e 6.

\begin{tabular}{|c|c|c|}
\cline { 2 - 3 } \multicolumn{1}{c|}{} & INSTANCIA 5 & INSTANCIA 6 \\
\cline { 2 - 3 } & Custo de Estocagem 1 & Custo de Estocagem 2 \\
\cline { 2 - 3 } & 5,1 & 15,1 \\
\cline { 2 - 3 } & 10,3 & 20,3 \\
\cline { 2 - 3 } & 10,5 & 30,5 \\
\cline { 2 - 3 } & 15,6 & 25,6 \\
\cline { 2 - 3 } & 45,8 & 55,8 \\
\cline { 2 - 3 } & 37,6 & 57,6 \\
\cline { 2 - 3 } & 18,1 & 28,1 \\
\cline { 2 - 3 } & 18,7 & 28,7 \\
\cline { 2 - 3 } & 32,7 & 62,7 \\
\hline Média Custo Estocagem & 15,8 & $\mathbf{4 1 , 0 2}$ \\
\hline Valor Otimo FO & $\mathbf{2 1 , 0 2}$ & $\mathbf{1 9 6 3 , 4 7 8}$ \\
\hline T resolução & $\mathbf{1 8 6 3 , 4 7 8}$ & $\mathbf{0 , 0 0 3}$ \\
\hline
\end{tabular}

Para verificar o impacto na alteração dos custos de movimentação, nas instâncias 7 e 8 , foram alterados os valores para cada tipo de embalagem. Desta forma, ao analisar a média dos custos de movimentação, na instância 8 , o custo é aumentado consideravelmente, resultando em um grande impacto na função objetivo, aumento de aproximadamente 1750 no valor ótimo da função objetivo, considerando um aumento médio de 27 do custo de movimentação.

Tabela 11. Resultado das análises das instâncias 7 e 8.

\begin{tabular}{|c|c|c|c|c|c|c|c|}
\hline & & & & & $\begin{array}{l}\text { Média Custo de } \\
\text { Movimentação }\end{array}$ & Valor Ótimo FO & T resolução \\
\hline INSTÂNCIA 7 & Custo de movimentação 1 & 35,2 & 58,4 & 60,7 & 51,43 & 4031,421 & 0,003 \\
\hline INSTÂNCIA 8 & Custo de movimentação 2 & 65,2 & 98,4 & 70,7 & 78,10 & 5779,114 & 0,001 \\
\hline
\end{tabular}

Por fim, as instâncias 9 e 10 foram propostas para analisar o comportamento do modelo matemático quando alterado a quantidade de embalagens disponíveis no circuito. Ao analisar a média de embalagens de cada uma das instâncias, quando há um aumento na quantidade disponível têm-se uma redução no valor ótimo da função objetivo. Essa relação pode ser explicada pelo aumento das possibilidades de alocação para cada tipo de peça, o que dependendo da escolha da embalagem, os custos finais são reduzidos.

Tabela 12. Resultado das análises das instâncias 9 e 10.

\begin{tabular}{|c|c|c|c|c|c|c|c|}
\hline & & & & & Média Qtd Embal. Disp. & Valor Ótimo FO & T resolução \\
\hline INSTÂNCIA 9 & Quantidade de Embalagens disponíveis 1 & 25 & 28 & 30 & 27,67 & 5909,434 & 0,002 \\
\hline INSTÁNCIA 10 & Quantidade de Embalagens disponíveis 2 & 85 & 98 & 50 & 77,67 & 5779,114 & 0,002 \\
\hline
\end{tabular}

As instâncias de 11 à 25, tiveram o intuito de verificar o comportamento do modelo quando inseridos dados muito pequenos ou muito grandes. Desta maneira, ao rodar com dados pequenos, a solução informada é inviável. Quando colocado em dados muito altos, observa-se que o custo final é aumentado consideravelmente a ponto de colocar em questão a real necessidade do uso dos três tipos de embalagens nos cenários rodados. Ou seja, faz-se necessário reavaliar 
os tipos de embalagens utilizados nos cenários, trazendo a possibilidade de ser inserida uma quarta embalagem no modelo, com características que melhor atenda ao fluxo logístico.

\section{CONCLUSÃo}

Ao analisar o cenário competitivo entre as empresas, todos os tipos de reduções de custos são importantes serem estudados. Desta forma, ao compreender o grande papel que uma embalagem tem dentro de um fluxo logístico, faz-se necessário estudos que auxiliem nos processos de definição do melhor tipo de embalagem para cada tipo de peça, a um menor custo possível.

Desta forma, o modelo proposto pelos autores Brizon et al (2004) auxiliou no processo de entendimento de como as definições de embalagens influenciam nos custos finais de projeto. Vale lembrar que em ambientes produtivos, cada peça tem uma embalagem definida, que caso seja mal projetada os custos de movimentação e de estocagem são elevados, aumentando os desperdícios de mão de obra, fluxos e estocagem.

Os resultados obtidos em cada uma das análises, especificamente para as 10 das 25 instâncias, foram satisfatórios uma vez que estão de acordo as características de problemas reais e atenderam a todas as restrições do modelo.

Como proposta futura, pretende-se criar um modelo matemático que englobe mais variáveis que são fundamentais no processo decisório do tipo da embalagem. Ou seja, agregar mais características realísticas tais como: criticidade da peça, ergonomia, rotas de abastecimento, métodos de abastecimentos, entre outras.

\section{REFERÊNCIAS BIBLIOGRÁFICAS}

Brizon, W. B. et al. 2004. Modelos matemáticos para a alocação de peças a embalagens no abastecimento de linhas de montagem. XXVI - Simpósio brasileiro de pesquisa operacional. São João Del - Rei/ MG.

Felippe, P. C. et al. 2005. Um modelo de programação matemática para alocação estática de caminhões visando ao atendimento de metas de produção e qualidade. REM: R. Esc. Minas, Ouro Preto, 58(1): 77-81, jan. mar.

Ianchan, R. et al. 2005. Um algoritmo genético híbrido para o planejamento de movimentação de gás da bacia de campos. XXVII Simpósio brasileiro de pesquisa Operacional. Gramados - RS.

Toso, R. J. 2007. Alguns aspectos da embalagem e a necessidade de integração do marketing com outras áreas da organização. Revista de Ciências Gerenciais, Vol 11, No 13.

Marques, Carlos E. C. 2009. A importância das embalagens na gestão da cadeia de abastecimento: caso prático. Universidade de Aveiro, departamento de Economia, Gestão e Engenharia industrial.

Moura, R. A.; Banzato J. M. 2000. Embalagem Unitização \& Conteinerização. IMAM, São Paulo.

Medeiros, J. L. et al. 2008. Um modelo de fluxo em rede para a logística de movimentação de contêineres. XL Simpósio brasileiro de pesquisa operacional. João pessoa - PB.

Nakamura, C. Y. 2010. Análise da viabilidade da utilização do transporte por cabotagem para a movimentação de automóveis no Brasil: um estudo de caso. Escola superior de agricultura "Luiz de Queiroz". Piracicaba.

Kussano, M. R.; Batalha, M. O. 2012. Custos logísticos agroindustriais: avaliação do escoamento da soja em grão do Mato Grosso para o mercado externo. Gest. Prod., São Carlos, v. 19, n. 3, p. 619-632.

Yamakami, A.; Ojima, A. L. R. 2006. Modelo de programação quadrática para análise da movimentação logística e comercialização da soja brasileira. Eng. Agríc., Jaboticabal, v.26, n.2, p.552-560, maio/ago.

Zeng, A. Z.; Rossetti, C. 2003. Developing a framework for evaluating the logistics cost in global sourcing process: an implementation and insigts. International Journal of Physical Distribution \& Logistics Management, v. 33, n. 9, p. 785-803. 


\title{
USE OF A MATHEMATICAL MODEL OF PIECES ALLOCATION FOR TESTS OVER DIFFERENT SCENARIOS
}

\author{
Tiago dos Santos Almeida, tiago_dosalmeida@hotmail.com \\ Helio Yochihiro Fuchigami, heliofuchigami@ yahoo.com.br² \\ Muris Lage Júnior, muris@ufg.br ${ }^{3}$ \\ Thiago Alves de Queiroz, taq@ufg.br ${ }^{4}$
}

\footnotetext{
${ }^{1}$ Federal University of Goiás- Department of Industrial Mathematics, Av. Dr. Lamartine Pinto de Avelar, 1120, University Sector. Scholarship from FAPEG.

${ }^{2}$ Federal University of Goiás- Department of Industrial Mathematics, Av. Dr. Lamartine Pinto de Avelar, 1120, University Sector

${ }^{3}$ Federal University of Goiás- Department of Industrial Mathematics, Av. Dr. Lamartine Pinto de Avelar, 1120, University Sector

${ }^{4}$ Federal University of Goiás- Department of Industrial Mathematics, Av. Dr. Lamartine Pinto de Avelar, 1120, University Sector
}

\begin{abstract}
Due to increased competition between companies, all costs involved in new projects have been taken into consideration in order to identify possible points of cost reduction. This paper aims to present a mathematical model and to perform various tests over different scenarios to study the impact in the optimal value of the objective function. Thus, each instance tested has the objective of point the model performance. We created 25 instances considering the stage of tests in order to study the solutions when varying the demand, cost allocation, cost of moving, the amount of packaging in the circuit and different groups of pieces. For the resolution of the model, we used the optimization solver Gurobi. The mathematical model aims to allocate pieces in packaging, so minimizing the total cost. The total cost involves handling and storage operations.
\end{abstract}

Keywords: storage, optimization, allocation and integer programming. 